\title{
Determination of Kerr and two-photon absorption coefficients of ABI thin films
}

\author{
Arturs Bundulis, Edgars Nitiss, Martins Rutkis \\ Institute of Solid State Physics, University of Latvia, Kengaraga 8, 1068 Riga, Latvia
}

\begin{abstract}
In this paper we report the results of studied nonlinear optical properties of DMABI-Ph6 in form of solutions with chloroform as solvent and guest-host thin films with poly(methyl methacrylate) as host material. We implemented the Zscan method for studies of Kerr and two-photon absorption of selected material. During experimental measurements we used $1064 \mathrm{~nm} \mathrm{Nd:YAG} \mathrm{laser} \mathrm{with} 30 \mathrm{ps}$ pulse duration and $10 \mathrm{~Hz}$ repetition rate. From acquired values of Kerr coefficients we calculated values for real part of third-order susceptibility, as well as second-order hyperpolarizability. Acquired data for DMABI-Ph6 were compared with data for other ABI derivatives studied previously to describe how different donor and acceptor groups influence third-order nonlinear optical properties.
\end{abstract}

Keywords: Kerr effect, two-photon absorption, organic materials, nonlinear optics, Z-scan, thin films.

\section{INTRODUCTION}

As the demand for bandwidth in telecommunication sector grows, it is essential to find new ways to increase the efficiency of telecommunication systems. One solution could be substituting existing hybrid system that employs electrical and optical signal with an all-optical communication system. To step towards this goal we need to design devices that can transfer, store and process optical information. While former of these devices has already been widely used in practical applications, both of the later ones are still in development phase. Devices for optical data storage ${ }^{1}$ and processing ${ }^{2}$ have been demonstrated in scientific papers, yet they are not efficient enough for practical applications. The key elements for optical data processing and storage are third-order nonlinear optical (NLO) materials that possess pronounced Kerr effect and two-photon absorption (TPA) ${ }^{3}$. Both effects manifest as optical property dependence on optical intensity, where Kerr effect characterises refractive index changes and TPA - absorption changes. Material selection process for these applications is based on various aspects ${ }^{4}$ : i) large molecular third-order NLO efficiency, ii) ultrafast NLO response time to acquire high bandwidth all-optical signal processing, iii) low optical losses due to absorption or scattering, iv) the possibility to retain microscopic NLO properties in macroscopic material forms. In the latter the main issue is evading any intermolecular interactions that could lead to decreasing of effective NLO properties of macroscopic material. v) Ability to process material in macroscopic form. One of the main advantages of organic materials for NLO applications is the possibility to tune the material properties by varying the molecular structure. Although vast amount of papers have been published regarding structural-property relations of organic materials ${ }^{5-7}$, we still do not possess enough information to carry out most of material selection process theoretically.

For this research we implemented Z-scan experimental method, as it is one of the most widely used methods for studies of Kerr and TPA effects ${ }^{8}$. Main advantages of Z-scan method compared to other methods for NLO studies (degenerated four-wave mixing $\operatorname{method}^{9}$, nonlinear ellipse rotation method ${ }^{10}$, Mach-Zehnder interferometer ${ }^{11}$ ) are that it is easy to assemble, you can study Kerr and TPA effects simultaneously as well as determine the sign of Kerr effect (for example degenerated four-wave mixing method can measure only the model of Kerr effect coefficient). As nonlinear refractive index changes can be induced by several different effects - third-order NLO, thermo-optical ${ }^{12}$, free charge carrier ${ }^{13}$ and other effects - it is essential to choose correct experimental conditions to separate only the specific effects, in this case third-order NLO ones. It is known, that inducing effects differ by response time ${ }^{8}$. As third-order NLO effects are of electronic origin, their response time is significantly faster compared to other effects and can be easily separated by choosing short pulse lasers with low pulse repetition rate.

In this paper we study 2-(4-(bis(5,5,5-triphenylpentyl)amino)benzylidene)-1H-indene-1,3(2H)-dione (DMABI-Ph6) in two forms. As thin films of guest-host system, where guest material is our organic compound and host is poly(methyl 
methacrylate) (PMMA) and as solutions with organic compound dissolved in chloroform. Because PMMA does not possess significant NLO properties it is suitable as host material ${ }^{14}$. Experimental measurements were carried out using $1064 \mathrm{~nm}$ Nd:YAG laser with 30 ps pulses at $10 \mathrm{~Hz}$ repetition rate (EKSPLA PL2140 Nd:YAG laser). Acquired results were compared with previously published results on third-order NLO properties of other aminobenziliden-1,3indandione $(\mathrm{ABI})$ derivatives in form of solution and results to better understand different donor and acceptor group influence on third-order NLO properties ${ }^{15}$.

\section{EXPERIMENTAL SECTION}

In this work we studied DMABI-Ph6 (see Figure 1) in form of thin films and solution. Thin film samples were prepared using spin-coating method. Firstly, PMMA and organic compound were dissolved in chloroform solution. Solution was spin-coated onto 1-inch square glass slide. Sample thickness was measured using a profilometer (Veeco Digital Instruments Dektak 150 Surface Profile Measurement System) and estimated to be d=1556 $\pm 34 \mathrm{~nm}$. Samples in solution form were prepared by dissolving organic compound in chloroform. Sample concentration was measured as organic compound mass over chloroform in percentage and varied from $0.003 \%$ to $0.8 \%$. This was done so sample would fit weak nonlinear media model implemented in data analysis process ${ }^{8}$. Studied samples were contained in $2 \mathrm{~mm}$ thick optical quartz cells.

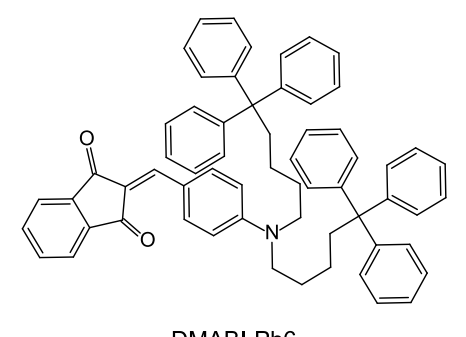

DMABI-Ph6

2-(4-(bis(5,5,5-triphenylpentyl)amino)benzylidene)$1 \mathrm{H}$-indene-1,3(2H)-dione

Figure 1: Name and structural formula of studied material.

Sample absorption spectrum was measures using Ocean Optics HR4000 Spectrometer. Acquired spectrum for DMABIPh6 dissolved in chloroform is shown in Figure 2 with peak at $493 \mathrm{~nm}$. From absorption spectrum it is evident that DMABi-Ph6 does not possess large absorption at $532 \mathrm{~nm}$ which suggests that TPA at 1064 nm should not be very strong ${ }^{15}$.

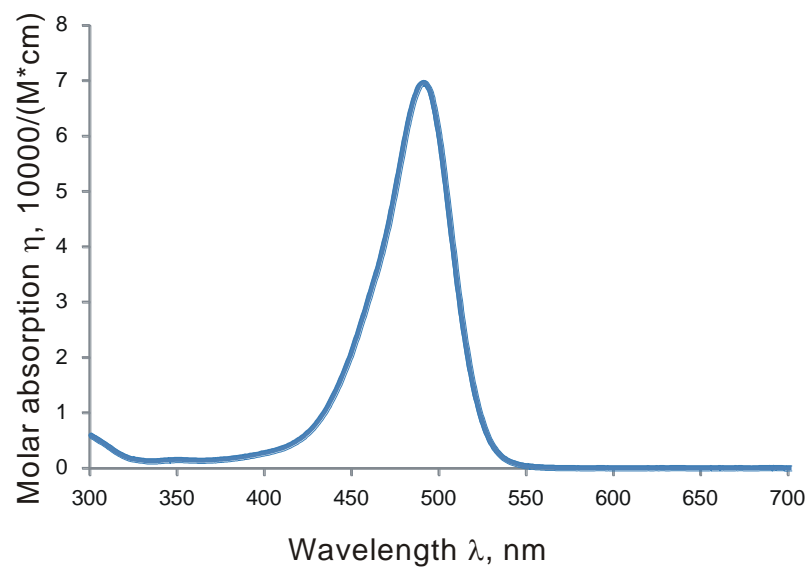

Figure 2: DMABI-Ph6 molar absorption spectrum. 
The description of Z-scan setup used in this work can be found in our previous paper ${ }^{15}$. For our experimental setup we used lens with a $11 \mathrm{~cm}$ focal length for laser beam focusing. Laser beam waist radius at focus was calculated to be $w_{0}=26$ $\mu \mathrm{m}$. For closed-aperture measurements we used $1 \mathrm{~mm}$ aperture which separated less than $1 \%$ of incident laser beam. Previously mentioned experimental parameters allow us to implement thin sample approximation for data processing ${ }^{8}$.

For description of Kerr and TPA effects we used the following formalism. Refractive index $n$ changes due to Kerr effect can be expressed in the following way:

$$
\mathrm{n}=\mathrm{n}_{0}+\mathrm{n}_{2} \mathrm{I} \text {, }
$$

where $n_{0}$ is linear refractive index of the material, $n_{2}$ is nonlinear refractive index and $I$ is incident light intensity. In case of TPA we study how materials absorption $\alpha$ changes due to incident light intensity:

$$
\alpha=\alpha_{0}+\alpha_{2} \mathrm{I}
$$

where $\alpha_{0}$ is linear absorption coefficient and $\alpha_{2}$ is nonlinear absorption coefficients.

One of the most essential aspects of Z-scan method is that one can study Kerr and TPA effects at the same time. This is done by measuring sample transmittance as function from sample position using two detectors - open aperture and closed aperture detectors. Open aperture measurement detects only TPA effect influence, while closed aperture detector measures both Kerr and TPA effects. If both of these effects are present in media, Kerr effect can be separated by dividing closed aperture data with open aperture data. If Kerr effect is present in NLO media, then sample transmittance can be expressed as follows:

$$
\mathrm{T}(\mathrm{z})=1+\frac{4 \Delta \Phi \frac{\mathrm{z}}{\mathrm{z}_{0}}}{\left(\frac{\mathrm{z}^{2}}{\mathrm{z}_{0}^{2}}+9\right)\left(\frac{\mathrm{z}^{2}}{\mathrm{z}_{0}^{2}}+1\right)},
$$

where $z$ is sample position relative to focal point and $\Delta \Phi$, and $z_{0}$ is parameters defined by following relations:

$$
\left\{\begin{array}{c}
\Delta \Phi=\mathrm{kn}_{2} \mathrm{IL}_{\mathrm{eff}} \\
\mathrm{L}_{\mathrm{eff}}=\frac{1-\mathrm{e}^{-\mathrm{\alpha L}}}{\alpha} \\
\mathrm{z}_{0}=\frac{\mathrm{kw}_{0}^{2}}{2}
\end{array}\right.
$$

where $w_{0}$ is beam waist radius at focal point, $L$ is sample thickness and $k$ is the wave number. This theoretical model can be applied for case of weak nonlinear media, for which $|\Delta \Phi|<\pi$. If TPA is present in NLO media, then sample transmittance can be expressed as follows:

$$
\mathrm{T}(\mathrm{z})=\sum_{\mathrm{i}=0}^{\infty} \frac{\left[\frac{\alpha_{2} \mathrm{~L}_{\text {eff }} \mathrm{I}}{\left(1+\frac{\mathrm{z}^{2}}{\mathrm{z}_{0}{ }^{2}}\right)}\right]^{\mathrm{m}}}{(\mathrm{m}+1)^{\frac{3}{2}}}
$$

Similar to Kerr effect this theoretical model can be used when weak nonlinear media criteria are meet $-\left|\alpha_{2} L_{e f f} I\right|<1$. This is essential for correct evaluation of NLO coefficients ${ }^{16}$. Laser intensity at focal point was varied in range from 10 to 100 $\mathrm{GW} / \mathrm{cm}^{2}$. Usage of higher laser intensities was limited by conditions for weak nonlinear media as well as to avoid sample degradation.

\section{RESAULTS AND DISCUSSION}

To calibrate our experimental setup, we performed a series of test measurements with $\mathrm{CS}_{2}$ and chloroform solutions as they are widely used as reference materials for third-order NLO studies. Acquired values of Kerr effect coefficient was $n_{2}=1.88 \pm 0.12 \cdot 10^{-15} \mathrm{~cm}^{2} / \mathrm{W}$ for chloroform and $n_{2}=3.10 \pm 0.25 \cdot 10^{-14} \mathrm{~cm}^{2} / \mathrm{W}$ for $\mathrm{CS}_{2}$. Both of these values fit the ones 
presented in literature ${ }^{8,17}$. Next we measured NLO properties of DMABI-Ph6 dissolved in chloroform. Experimental data are shown in Figure 3. As no transmittance changes was observed for open aperture signal, we conclude that DMABIPh6 possess no significant TPA effect. As solutions and solutes contribution to overall NLO properties of sample is defined by weight fraction, we derived Kerr effect coefficient of DMABI-Ph6 using following equation ${ }^{18}$ :

$$
\mathrm{n}_{2}=(1-\rho) \mathrm{n}_{2 ; \mathrm{CHCl}_{3}}+\rho \mathrm{n}_{2 ; \text { solute }}
$$

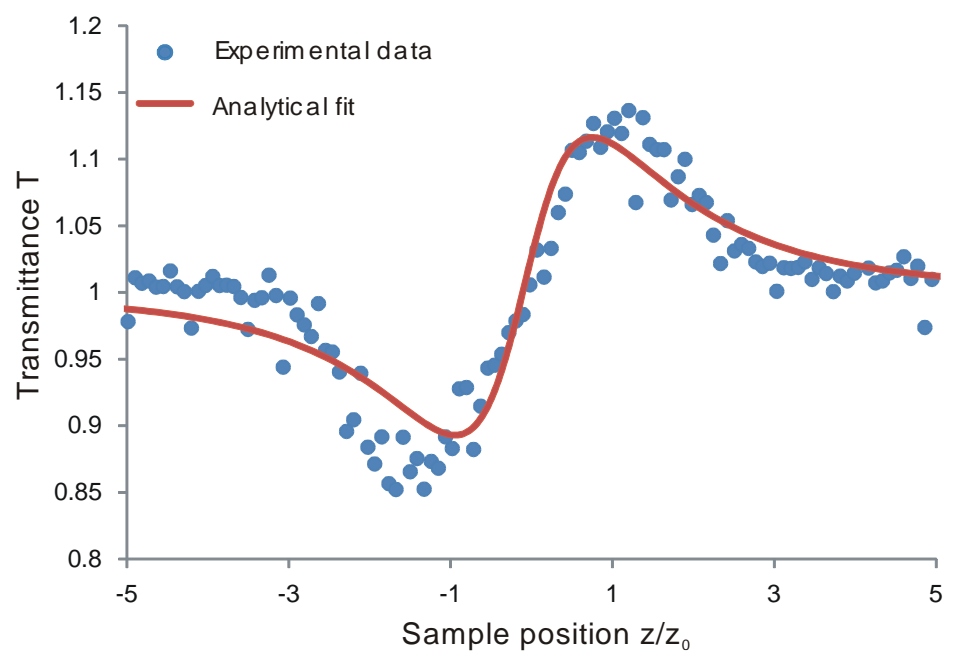

Figure 3: Experimental data for DMABI-Ph6 dissolved in chloroform. Sample concentration in this measurement was $0,22 \%$ and laser pulse irradiance was approximately $31 \mathrm{GW} / \mathrm{cm}^{2}$.

From experimental data we calculated that Kerr coefficient of DMABI-Ph6 is $n_{2}=-1.05 \pm 0.11 \cdot 10^{-13} \mathrm{~cm}^{2} / \mathrm{W}$. Similar as for solution measurements, before studying organic compounds in form of thin films, we measured NLO properties of pure PMMA thin film for reference purposes. As we did not observe any transmittance changes in open and closed aperture measurements we concluded that PMMA influence on refractive index and absorption changes is negligible. Experimental data for guest-host thin films containing DMABI-Ph6 as guest material can be seen in Figure 4. From experimental data, we obtained that DMABI-Ph6 in thin film configuration possess Kerr coefficient $n_{2}=-8.07 \pm 0.56 \cdot 10^{-12}$ $\mathrm{cm}^{2} / \mathrm{W}$. From this we can see that Kerr coefficient is significantly larger when material is prepared in form of thin films.

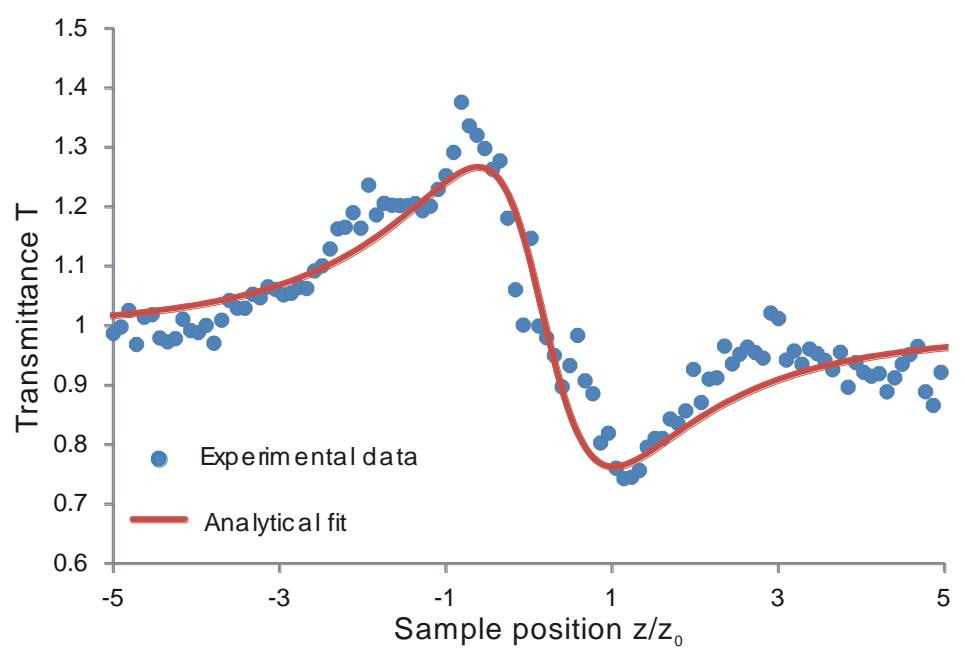

Figure 4: Experimental data for DMABI-Ph6 thin film. Laser pulse irradiance at focal point was approximately $23 \mathrm{GW} / \mathrm{cm}^{2}$. 
From values of Kerr and TFA coefficients we calculated values for real and imaginary parts of third-order susceptibility of our materials using formulas presented in literature ${ }^{19}$ :

$$
\chi_{\operatorname{Re}}^{(3)}=\left(4 \cdot \frac{\varepsilon_{0} \cdot c \cdot n_{0}^{2}}{3}\right) \cdot \mathrm{n}_{2}\left(\frac{\mathrm{m}^{2}}{\mathrm{~W}}\right)
$$

where $\varepsilon_{0}$ is vacuum dielectric constant, $c$ is speed of light in vacuum and $\lambda$ is laser wavelength. Third-order susceptibility characterizes material properties on macroscopic scale. To compare NLO properties of single molecule we used values for second-order hyperpolarizability of our materials ${ }^{20}$ :

$$
\gamma=\frac{\chi^{(3)}}{\left[\frac{1}{3}\left(\mathrm{n}_{0}^{2}+2\right)\right]^{4} \mathrm{~N}},
$$

where $N$ is molecule concentration per $\mathrm{cm}^{3}$. Acquired values for NLO coefficients are presented in Table 2 . By comparing these results to previously obtained for other ABI derivatives in form of solutions, we can conclude that DMABI-Ph6 possess smaller Kerr coefficient, but higher second-order hyperpolarizability then other ABI derivatives. From this it is evident that triphenylpentyl groups added to donor part of molecule has small contribution to overall NLO properties of molecule. At the same time it is important to emphasize that triphenylpentyl groups limits the probability of crystallization and can lead to formation of higher concentration thin films ${ }^{21}$. Other ABI derivative concentration is limited due to crystallization at high concentrations ${ }^{22}$.

\begin{tabular}{|c|c|c|c|c|c|c|c|c|}
\hline Material & $\begin{array}{l}\text { Extinction } \\
\text { coefficient } \\
\text { at } 532 \mathrm{~nm}\end{array}$ & $\begin{array}{c}\mathrm{M}, \\
\mathrm{g} / \mathrm{mol}\end{array}$ & $\begin{array}{c}\mathrm{n}_{2 ; \text { Kerr, }} \mathrm{cm}^{2} / \mathrm{W} \cdot \\
10^{-13}(\mathrm{~S})\end{array}$ & $\begin{array}{c}\mathrm{n}_{2 ; \mathrm{Kerr}}, \mathrm{cm}^{2} / \mathrm{W} \\
\cdot 10^{-12}(\mathrm{TF})\end{array}$ & $\begin{array}{c}\chi \mathrm{Re}, \\
\mathrm{m}^{2} / \mathrm{V}^{2} \cdot 10^{-19} \\
(\mathrm{~S})\end{array}$ & $\begin{array}{c}\chi \mathrm{Re}, \\
\mathrm{m}^{2} / \mathrm{V}^{2} \cdot 10^{-18} \\
(\mathrm{TF})\end{array}$ & $\begin{array}{l}\gamma, \text { esu } \cdot 10- \\
{ }_{34}(\mathrm{~S})\end{array}$ & $\begin{array}{c}\gamma, \text { esu } \cdot 10-^{34} \\
(\mathrm{TF})\end{array}$ \\
\hline $\mathrm{Ph} 6$ & 3577 & 846.13 & $-1.05 \pm 0.11$ & $-8.07 \pm 0.56$ & $-1.09 \pm 0.11$ & $-8.38 \pm 0.58$ & $-14.1 \pm 1.5$ & $-10.81 \pm 0.75$ \\
\hline
\end{tabular}

Table 1. Nonlinear optical properties of DMABI-Ph6 in form of solution (S) and thin films (TF).

\section{CONCLUSIONS}

In this paper we studied NLO effects of DMABI-Ph6 in chloroform solution and guest-host thin films with PMMA as host material. By comparing results of both sample classes, we observed higher values for thin-film samples. During experimental measurements we concluded that DMABI-Ph6 possess no significant two-photon absorption at $1064 \mathrm{~nm}$. In comparison to other ABI derivatives DMABI-Ph6 possess smaller overall induced refractive index changes but larger second-order hyperpolarizability value. This is due to low NLO contribution of triphenylpentyl groups. Although these molecular alternations decrease Kerr coefficient value, it allows for formation of higher concentration thin films, which is essential for practical NLO applications.

\section{ACKNOWLEDGMENT}

This material is based upon work supported by the ERDF 1.1.1.1 activity project Nr. 1.1.1.1/16/A/046 "Application assessment of novel organic materials by prototyping of photonic devices"

\section{REFERENCES}

[1] Hu, D., Hu, Y., Huang, W.., Zhang, Q., “Two-photon induced data storage in hydrogen bonded supramolecular azopolymers," Opt. Commun. 285(24), 4941-4945, Elsevier (2012).

[2] Ahadi, S.., Granpayeh, N., "Femtosecond all-optical switching based on asymmetric plasmonic Kerr Fabry-Perot 
resonators," Opt. Commun. 349, 36-41, Elsevier (2015).

[3] Gu, B., Zhao, C., Baev, A., Yong, K.-T.., Prasad, P. N., "Molecular nonlinear optics: recent advances and applications," Adv. Opt. Photonics 8(2), 328 (2016).

[4] Hales, J. M., Barlow, S., Kim, H., Mukhopadhyay, S., Brédas, J.-L., Perry, J. W.., Marder, S. R., "Design of Organic Chromophores for All-Optical Signal Processing Applications," Chem. Mater. 26(1), 549-560 (2014).

[5] Webster, S., Fu, J., Padilha, L. A., Przhonska, O. V., Hagan, D. J., Van Stryland, E. W., Bondar, M. V., Slominsky, Y. L.., Kachkovski, A. D., "Comparison of nonlinear absorption in three similar dyes: Polymethine, squaraine and tetraone," Chem. Phys. 348(1-3), 143-151 (2008).

[6] Fu, J., Padilha, L. A., Hagan, D. J., Van Stryland, E. W., Przhonska, O. V., Bondar, M. V., Slominsky, Y. L.., Kachkovski, A. D., "Molecular structure-two-photon absorption property relations in polymethine dyes," J. Opt. Soc. Am. B 24(1), 56 (2007).

[7] Lim, J. H., Przhonska, O. V., Khodja, S., Yang, S., Ross, T. S., Hagan, D. J., Van Stryland, E. W., Bondar, M. V., Slominsky, Y. L., "Polymethine and squarylium molecules with large excited-state absorption," Chem. Phys. 245(1-3), 79-97 (1999).

[8] Sheik-Bahae, M., Said, A. A., Wei, T.-H., Hagan, D. J.,, Van Stryland, E. W., "Sensitive measurement of optical nonlinearities using a single beam," IEEE J. Quantum Electron. 26(4), 760-769 (1990).

[9] Kityk, I. V., Fahmi, A., Sahraoui, B., Rivoire, G.., Feeks, I., "Nitrobenzene as a material for the fast-respond degenerate four-wave mixing," Opt. Mater. (Amst). 16(4), 417-429 (2001).

[10] Liu, Z., Yan, X., Tian, J., Zhou, W.., Zang, W., "Nonlinear ellipse rotation modified Z-scan measurements of third-order nonlinear susceptibility tensor," Opt. Express 15(20), 13351 (2007).

[11] Bundulis, A., Nitiss, E., Busenbergs, J.., Rutkis, M., "Mach-Zehnder interferometer implementation for thermooptical and Kerr effect study," Appl. Phys. B 124(4), 56 (2018).

[12] Gnoli, A., Razzari, L.., Righini, M., "Z-scan measurements using high repetition rate lasers: how to manage thermal effects," Opt. Express 13(20), 7976 (2005).

[13] Said, A. A., Sheik-Bahae, M., Hagan, D. J., Wei, T. H., Wang, J., Young, J.., Van Stryland, E. W., "Determination of bound-electronic and free-carrier nonlinearities in ZnSe, GaAs, CdTe, and ZnTe," J. Opt. Soc. Am. B 9(3), 405 (1992).

[14] Poornesh, P., Hegde, P. K., Umesh, G., Manjunatha, M. G., Manjunatha, K. B.., Adhikari, A. V., "Nonlinear optical and optical power limiting studies on a new thiophene-based conjugated polymer in solution and solid PMMA matrix," Opt. Laser Technol. 42(1), 230-236, Elsevier (2010).

[15] Bundulis, A., Nitiss, E., Mihailovs, I., Busenbergs, J.., Rutkis, M., "Study of Structure-Third-Order Susceptibility Relation of Indandione Derivatives,” J. Phys. Chem. C 120(48), 27515-27522 (2016).

[16] Ajami, A., Gruber, P., Tromayer, M., Husinsky, W., Stampfl, J., Liska, R.., Ovsianikov, A., "Evidence of concentration dependence of the two-photon absorption cross section: Determining the 'true' cross section value,” Opt. Mater. (Amst). 47, 524-529, Elsevier B.V. (2015).

[17] Cassano, T., Tommasi, R., Ferrara, M., Babudri, F., Farinola, G. M.., Naso, F., "Substituent-dependence of the optical nonlinearities in poly(2,5-dialkoxy-p-phenylenevinylene) polymers investigated by the Z-scan technique," Chem. Phys. 272(1), 111-118 (2001).

[18] Samoc, M., Samoc, A., Luther-Davies, B., Bao, Z., Yu, L., Hsieh, B.., Scherf, U., "Femtosecond Z-scan and degenerate four-wave mixing measurements of real and imaginary parts of the third-order nonlinearity of soluble 
conjugated polymers,” J. Opt. Soc. Am. B 15(2), 817 (1998).

[19] del Coso, R.., Solis, J., "Relation between nonlinear refractive index and third-order susceptibility in absorbing media,” J. Opt. Soc. Am. B 21(3), 640 (2004).

[20] Sharafudeen, K. N., Adithya, A., Vijayakumar, S., Sudheesh, P., Kalluraya, B.., Chandrasekharan, K., "Multiphoton absorption process and self-focusing effect in coumarin derivative doped PMMA films by z-scan and optical limiting studies," Curr. Appl. Phys. 11(4), 1089-1093, Elsevier B.V (2011).

[21] Nitiss, E., Tokmakovs, A., Pudzs, K., Busenbergs, J.., Rutkis, M., "All-organic electro-optic waveguide modulator comprising SU-8 and nonlinear optical polymer," Opt. Express 25(25), 31036 (2017).

[22] Rutkis, M., Vembris, A., Zauls, V., Tokmakovs, A., Fonavs, E., Jurgis, A.., Kampars, V., "Novel second-order nonlinear optical polymer materials containing indandione derivativatives as a chromophore," Org. Optoelectron. Photonics II, P. L. Heremans, M. Muccini, and E. A. Meulenkamp, Eds., 61922Q (2006). 